\title{
Efecto de las cargas articulares sobre el flujo y pH salival
}

\author{
Effect of TMJ loading on salivary flow and $\mathrm{pH}$
}

\author{
Castro RJ', Bravo C², Alcaino V², Giacaman RA ${ }^{3}$
}

\begin{abstract}
RESUMEN
Una alteración del flujo salival es clave en el desarrollo de caries, enfermedad periodontal e infecciones oportunistas. El flujo salival está determinado por diversos estímulos que actúan sobre receptores de distinta naturaleza, entre ellos mecanoreceptores articulares. Algunos estudios demuestran que las cargas articulares actúan sobre estos receptores, modificando cualitativa y cuantitativamente la secreción salival. El objetivo de este estudio fue determinar si existe una relación entre la aplicación controlada de cargas articulares y cambios en el flujo y pH salival. 30 voluntarios fueron seleccionados según criterios de inclusión establecidos. Se elaboró un dispositivo interoclusal que en relación a piezas 1.5 y 2.5 , tenía botones acrílicos de $1 \mathrm{~mm}$ de espesor, los que al ejercer fuerzas sobre ellos provocan cargas reversibles sobre la ATM. Antes de usar el dispositivo, en cada individuo se midió flujo salival no estimulado (FSNE) el flujo salival estimulado (FSE) y el pH de ambos. Tras un periodo de adaptación diaria por una semana, cada participante usó el dispositivo durante 1 hora, ejerciendo fuerza masticatoria sostenida. Luego se midieron ambos flujos y el pH correspondiente. El procedimiento fue repetido por 7 días, bajo supervisión. No se observó una relación aparente entre la aplicación controlada de cargas articulares y cambios volumétricos de flujo salival. Las mujeres tuvieron un FSE y FSNE menor al de los hombres. La aplicación de sobrecargas articulares por un breve período de tiempo provoca una caída del pH del FSE luego de 3 días de aplicación.
\end{abstract}

Rev. Clin. Periodoncia Implantol. Rehabil. Oral Vol. 4(1); 13-16, 2011.

Palabras clave: Flujo salival estimulado, flujo salival no estimulado, cargas articulares.

\section{ABSTRACT}

Altered salivary flow is key for the onset of dental caries, periodontal disease and opportunistic infections. Salivary flow is determined by various stimuli on several receptors, including joint mechanoreceptors. Some studies show that loads on the TMJ affect these receptors causing changes on the quality and quantity of saliva secretion. The aim of this study was to determine whether a relation exists between controlled load application and changes on salivary flow and $\mathrm{pH}$. Thirty volunteer subjects were recruited after excluding by inclusion criteria. A total coverage intraoral appliance was made with $1 \mathrm{~mm}$ acrylic buttons on tooth 1.5 and 2.5. The buttons were intentioned to direct the force vectors to apply a reversible load on the TMJ. Before using it, the Unstimulated salivary flow (USF), the Stimulated salivary flow (SSF) and the pH from each flow was obtained from each subject. After an adaptation period of a day, each volunteer used the appliance exerting steady moderate loads 1 hour/day for one week. Under the supervision of the examiner, the USF, SSF and both $\mathrm{pH}$ were daily registered for the entire week. There was not an apparent association between applying controlled loads on the TMJ and volumetric changes of the salivary flow. Women, however, showed lower USF and SSF than men. Load application through an oral appliance is associated with SSF pH drop from the third day.

Rev. Clin. Periodoncia Implantol. Rehabil. Oral Vol. 4(1); 13-16, 2011.

Key words: Unstimulated salivary flow, stimulated salivary flow, salivary flow pH, joint loads.

\section{INTRODUCCIÓN}

La saliva juega un significativo rol en la preservación y mantención de la salud oral ${ }^{(1)}$. Diversos autores han indicado que una alteración del flujo salival es un factor clave en el desarrollo de caries, enfermedad periodontal e infecciones oportunistas ${ }^{(2)}$. Además, una inadecuada función glandular de la secreción salival provoca un deterioro en el proceso de alimentación, dificultando la masticación, el paso de los alimentos al tracto digestivo, e incluso produce modificaciones en el habla ${ }^{(3)}$.

El flujo salival está determinado por diversos estímulos que actúan sobre receptores de distinta naturaleza: gustativos, olfativos, nociceptores y mecanoreceptores articulares ${ }^{(4)}$. Éstos últimos son estimulados durante el proceso de masticación ${ }^{(3)}$ y esta información se alteraría bajo ciertas patologías, como por ejemplo las sobrecargas que originan Trastornos Temporomandibulares $(\text { TTM })^{(5)}$. Dado lo anterior, resulta relevante el estudiar el comportamiento de estos receptores al ser sometidos a cargas articulares repetitivas, reconocido factor de iniciación de $\operatorname{TTM}^{(6)}$.

Algunos estudios demuestran que las cargas articulares nocivas provocan cambios en la integridad de la salud oral, alterando el ciclo biomecánico funcional del sistema orofacial, específicamente sobre los receptores encargados del reflejo de la salivación ${ }^{(7)}$. De este modo, alteraciones de distinta índole que afecten la integridad de los receptores pueden gatillar cambios cualitativos y cuantitativos en la secreción salival(8).

El pH salival, por su parte, crea condiciones ecológicas bucales que mantienen el equilibrio medioambiental previniendo la aparición de patologías como la caries dental. Sin embargo, no se han descrito alteraciones del $\mathrm{pH}$ asociadas a cargas articulares excesivas o durante los TTM. Existe una relación reportada entre el flujo salival y el $\mathrm{pH}$ de la saliva $^{(9)}$ debido a las variaciones en las concentraciones de bicarbonato y fosfato asociadas con los cambios volumétricos. De esta forma, se puede especular que alteraciones del flujo salival repercutieran en el $\mathrm{pH}$, de existir una asociación funcional entre los receptores articulares y los que regulan el flujo de saliva a nivel glandular.

El hallazgo de una relación entre TTM y alteraciones del flujo salival contribuiría al conocimiento de la integración funcional de los diversos componentes de la unidad cráneocervical y expandiría el espectro de los alcances a los que pueden dar origen las patologías funcionales del sistema. El objetivo de este estudio, por lo tanto, fue determinar si existe una relación entre la aplicación controlada de cargas articulares y cambios en el flujo y $\mathrm{pH}$ salival. 


\section{PACIENTES Y MÉTODOS}

Se invitó a participar a 30 individuos, de entre 20 a 27 años de edad, 15 hombres y 15 mujeres. Todos los participantes eran alumnos de la Carrera de Odontología de la Universidad de Talca, Chile. Para ingresar al estudio, los sujetos debían leer y firmar un consentimiento informado. El protocolo del estudio fue aprobado por el Comité de Bioética de la Universidad de Talca.

Los voluntarios que presentaron signos o síntomas de TTM ${ }^{(10)}$, movilidad dentaria patológica, tratamiento de ortodoncia actual, consumo de fármacos de cualquier tipo o enfermedades que alteraran la secreción salival fueron excluidos del estudio. A cada participante se le realizó una anamnesis general, examen clínico extra e intraoral y los datos fueron registrados en una Ficha Clínica confeccionada para el estudio.

Se tomaron impresiones del maxilar con hidrocoloide irreversible tipo II regular (Alginato, Jeltrate Dustless, DENTSPLY Caulk, Milford) y se confeccionaron modelos de yeso piedra. Sobre estos, con una lámina de acetato de celulosa rígida (Clear Treatment Splint Sheets. 5" x 5". Ultradent Products Inc.) de un $1 \mathrm{~mm}$ de espesor, se elaboró un dispositivo interoclusal similar a un plano Michigan, mediante un aparato conformador de acetato al vacío (Econo Vac, Model 80187. Buffalo Dental. N.Y.).

En relación a las piezas 1.5 y 2.5 , se aumentó la altura del dispositivo mediante dos botones de acrílico de autopolimerización de $5 \mathrm{~mm}$ de diámetro y $1 \mathrm{~mm}$ de espesor. De esta manera el paciente al ejercer fuerza masticatoria sobre éste, los vectores de fuerza hacia arriba y adelante de los músculos maseteros, temporal y pterigoideo medial, generan una carga reversible sobre la ATM $^{(11)}$.

Confeccionado el dispositivo y antes de su uso, en cada individuo se midió el flujo salival no Estimulado y Estimulado, usando el protocolo del Departamento de Cariología de la Universidad de Malmo, Suecia ${ }^{(12)}$ y clasificado según el programa Cariograma ${ }^{(13)}$. Inmediatamente obtenida la muestra salival, se obtuvo un valor de $\mathrm{pH}$ utilizando un electrodo $(\mathrm{pH}$ electrode $0-14 \mathrm{pH}, 0-90^{\circ} \mathrm{C}-\mathrm{PE}-149 \mathrm{G}$ ), conectado a un potenciómetro (GOnDO Bench Top pH-mV-Temperature Meter - PL-600Lab) calibrado al inicio de cada sesión, usando las soluciones estándar de $\mathrm{pH} 4.0$ y 7.0 .

Cada participante utilizó el dispositivo interoclusal por periodos cortos, no mayor a 30 minutos con intervalos de 3 horas durante la primera semana de ensayo, con el fin de adaptar al paciente al uso confortable del dispositivo. Pasada la semana de adaptación, los individuos usaron el dispositivo durante 1 hora, con la instrucción de ejercer una fuerza masticatoria sostenida sobre él, para que se produzca una carga articular ${ }^{(11)}$. Pasada la hora de uso se midieron ambos flujos salivales y el pH de ambos. Este procedimiento se repitió durante 7 días consecutivos siguiendo el mismo protocolo bajo la constante supervisión del operador. Las mediciones fueron todos los días a la misma hora entre las 10 y 15 horas, al menos 60 minutos después de haber consumido alimentos $^{(12)}$. Se evaluó, durante todo el periodo de ensayo, la aparición de signos y síntomas de TTM. En caso de presentarse, el sujeto debía ser excluido inmediatamente del estudio y el dispositivo regresado a los investigadores. Del mismo modo, al término del estudio a todos los participantes les fue retirado el dispositivo con el fin de evitar su uso inadecuado.

Los datos obtenidos fueron analizados con el test Kolmogorov - Smirnov el que indicó que presentan una distribución normal. Para comparar las diferencias entre los días de uso del dispositivo mediante el test ANOVA seguido de un análisis post-hoc con el test Tukey. Para comparar las diferencias producidas por sexo se utilizó el test t de Student. Se consideraron significativas estadísticamente las diferencias si el valor $p<0.05$. Los análisis fueron efectuados a través de un software estadístico (SPSS, Statistical Package for the Social Sciences $\odot$, 17.0 para Windows).

\section{RESULTADOS}

\section{Flujo Salival No Estimulado}

Los resultados para el Flujo Salival No Estimulado (FSNE) del total de la muestra y según género, se presentan en la Figura 1. La media del flujo salival No Estimulado para el total de los sujetos estudiados fue de $6.7 \mathrm{ml} / \mathrm{min}$ (S.D. 2.7). Al realizar el análisis comparativo entre el promedio del FSNE sin intervención y los promedios de cada día de intervención, no se encontraron diferencias estadísticamente significativas $(p>0.05)$. Al analizar los valores según género, el promedio del FSNE para el grupo de mujeres fue de $6.3 \mathrm{ml} / \mathrm{min}$ (S.D. 2.7), el que fue menor que el de los hombres $(7.2 \mathrm{ml} / \mathrm{min}$; S.D. 2.6) $(p<0.0001)$.

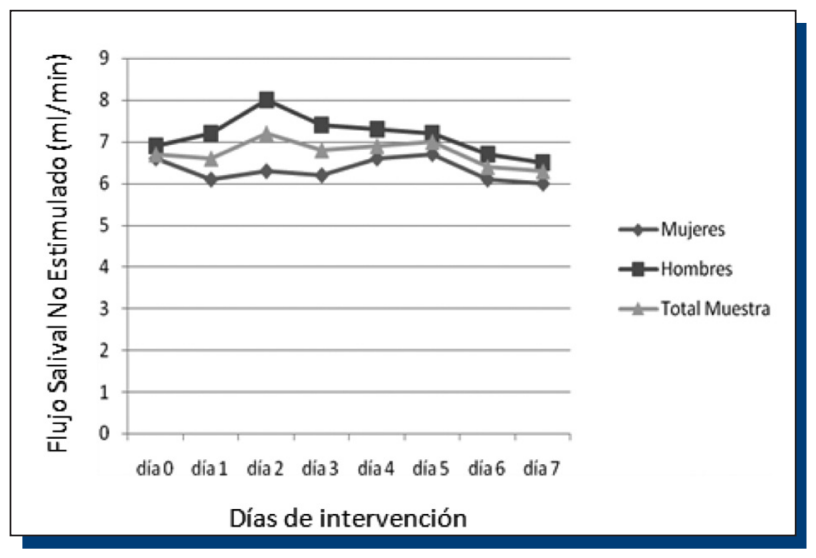

Figura 1. FSNE del total de la muestra y según género.

\section{Flujo Salival Estimulado}

Los resultados para el Flujo Salival Estimulado (FSE) del total de la muestra y según género, se presentan en la Figura 2. El promedio del FSE para el total de los sujetos estudiados fue $7.7 \mathrm{ml} / \mathrm{min}$ (S.D. 3.4). No se observaron diferencias entre el promedio del FSE sin intervención y el de cada día de intervención ( $p>0.05$ ). Al igual que en el FSNE, las mujeres del estudio mostraron valores más bajos de FSE $(7.3 \mathrm{ml} / \mathrm{min}$; S.D. 3.1) que los hombres $(8.1 \mathrm{ml} / \mathrm{min}$; S.D. 3.7) $(p<0.0001)$

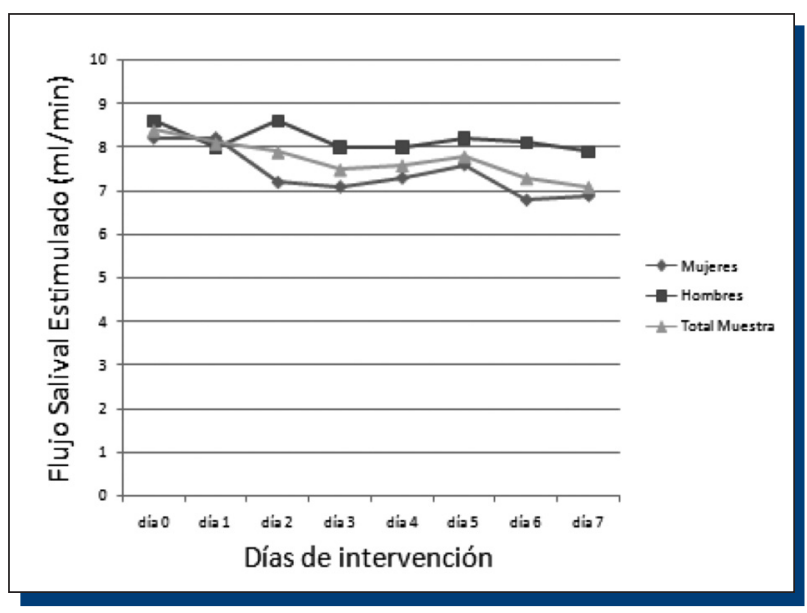

Figura 2. FSE del total de la muestra y según género.

\section{pH de la Muestra de Flujo Salival No Estimulado}

Los resultados para el pH de la muestra de Flujo Salival No Estimulado (pHFSNE) del total de la muestra y según género, se presentan en la Figura 3. El promedio del pHFSNE para el total de los sujetos estudiados, considerando el registro de los siete días de intervención, fue 5.2 (S.D. 0.5). No se detectaron diferencias significativas entre el inicio del estudio y los valores de pHFSNE de cada día de aplicación de cargas mediante los aparatos intraorales $(p>0.05)$. Los valores de pHFSNE fueron menores en las muestras obtenidas de mujeres que de los hombres $(p<0.0001)$ (Figura 3$)$. 


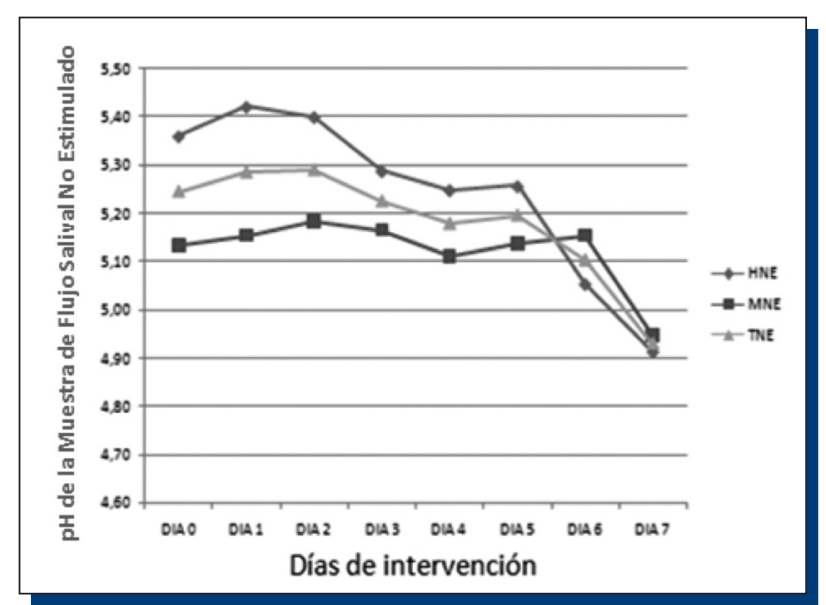

Figura 3. pHFSNE del total de la muestra y según género.

\section{pH de la Muestra de Flujo Salival Estimulado}

Los resultados del pH de la muestra de Flujo Salival Estimulado (PhFSE) del total de la muestra y según género, se presentan en la Figura 4. El valor promedio de pHFSE de todos los sujetos, considerando el registro de los siete días de intervención, fue 5.9 (S.D. 0.5). A contar del tercer día de aplicación de cargas mediante los dispositivos intraorales, se constató una disminución del pHFSE $(p<0.05)$. De manera similar a lo observado para el pHFSNE, las mujeres mostraron menor pHFSE que los hombres $(p<0.0001)$ (Figura 4).

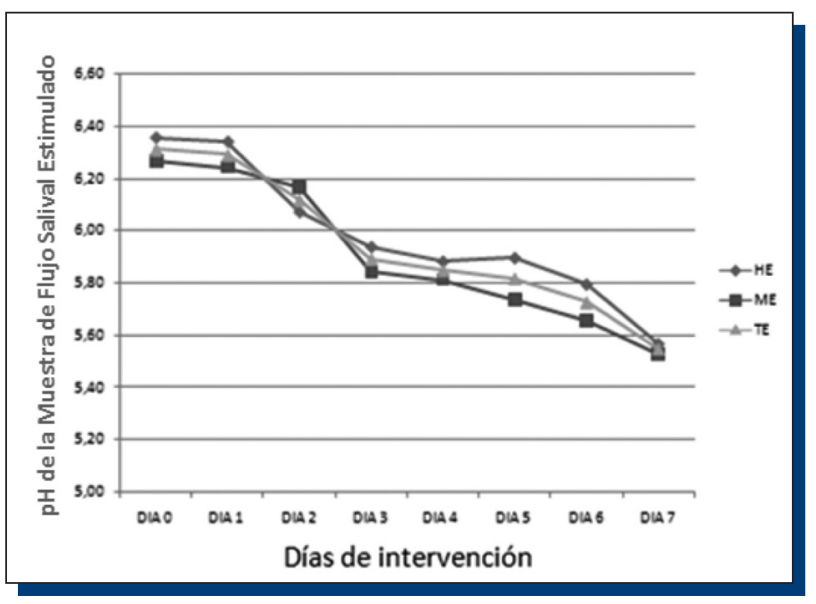

Figura 4. pHFSE del total de la muestra y según género.

\section{DISCUSIÓN}

El flujo salival está controlado por múltiples factores ${ }^{(14)}$. Los resultados obtenidos en este estudio pueden haber sido afectados por algunos de ellos y por ende, influenciado los valores obtenidos. Por ejemplo, el método de la estimulación salival mecánica mediante la masticación es el de mayor uso para el estudio del flujo salival(12). Sin embargo, en el presente estudio el haber al utilizado parafina vegetal, inerte y sin sabor, no estimula los receptores gustativos y olfativos, lo que podría influenciar negativamente el volumen salival obtenido. Además, la frecuencia de masticación y fuerza de apriete dentario de cada individuo no fue controlada, ambos importantes factores que intervienen directamente en los valores de flujo Estimulado.

Todos los valores obtenidos para FSNE y FSE sin intervención, están dentro de los rangos de normalidad según la clasificación de flujo salival entregada por Cariograma ${ }^{(13)}$. Contrariamente, los datos de $\mathrm{pH}$ obtenidos de las muestras de ambos flujos fueron menores que el rango normal ${ }^{(9)}$, especialmente el $\mathrm{pH}$ de la muestra de FSNE (promedio 5.2; S.D. 0.5). Pese a que se ha reportado que la saliva tiende a mantener la neutralidad del $\mathrm{pH}$ dada su capacidad tamponante ${ }^{(9)}$, en este estudio los valores de $\mathrm{pH}$ disminuyen con la aplicación de cargas. No es posible explicar el mecanismo que interviene en este proceso, lo que sugiere la necesidad de profundizar en esta área de investigación. En cuanto al pHFSE, se observó una disminución sostenida y significativa en los valores desde el tercer día de intervención para ambos géneros. Estos resultados, atribuibles a la carga que se generó sobre las ATM durante los días de estudio, no pueden ser contrastados con otras investigaciones debido a que no existen reportes previos que relacionen las cargas articulares con alguna alteración del $\mathrm{pH}$ salival. Lo anterior resulta interesante, ya que se trabajó con una muestra compuesta por individuos jóvenes, sanos y sin consumo de fármacos que pudiesen haber alterado cualitativa o cuantitativamente el flujo salival. Este hallazgo requiere mayor investigación en futuros estudios.

No obstante presentar un leve descenso en los valores de FSE y FSNE, estos no fueron estadísticamente significativos, lo que sugiere que sobrecargas moderadas a nivel articular no afectan el volumen del FSNE, lo que concuerda con algunos autores que describen que este, depende de la correcta función glandular ${ }^{(2,15)}$ y no se ve afectado por alteraciones en la estimulación mecánica de los receptores. En caso del FSE, la disminución observada a medida que transcurrieron los días de estudio es leve y no significativa. Condiciones experimentales diferentes o un mayor número de sujetos podrían haber entregado cambios más ostensibles, lo que concordaría con estudios previos ${ }^{(16)}$, los que señalan que el FSE va disminuyendo, asociándose al envío de señales perturbadas por parte de los mecanoreceptores articulares. Esta perturbación en el envió de señales se debe al proceso de masticación, principal estimulo de la saliva estimulada, en ausencia de estímulos gustativos ${ }^{(17)}$, el que es alterado por las cargas articulares ${ }^{(18)}$. Un factor clave para la interpretación de los resultados es el papel que podrían jugar los receptores de la mucosa oral, así como las glándulas salivales menores (dispersas por toda la cavidad bucal), manteniendo el equilibrio y un normal volumen de saliva, a pesar de la estimulación anómala de los receptores articulares.

Al realizar la comparación de valores obtenidos para flujo y $\mathrm{pH}$ (estimulado y no estimulado) entre hombres y mujeres, se encuentran diferencias significativas. Esto podría deberse a que junto a la alteración de los mecanoreceptores encargados del reflejo de la salivación, otras alteraciones o condiciones ligadas al género, como factores sicológicos $u$ hormonales entrarían en juego. Lo anterior no concuerda con estudios previos $^{(19)}$ los que señalan que la estimulación de los mecanoreceptores está asociada con un decline del FSE, independiente del género, edad y medicación de los pacientes.

El diseño del estudio se realiza con sujetos sanos y en un corto plazo. Resultaría interesante analizar que sucede con el flujo salival en aquellos individuos sometidos a una sobrecarga articular crónica, como es el caso de pacientes con TTM o parafunciones de larga data. Con respecto a lo anterior, la literatura sólo describe una relación entre TTM e hiposalivación en individuos que padecen artritis reumatoídea ${ }^{(15)}$. Pese a lo breve de la intervención, es importante señalar que el uso de un dispositivo interoclusal, similar a una férula oclusal, no causa alteraciones significativas en el volumen salival, al menos durante el periodo evaluado.

En conclusión, no existe una relación aparente entre la aplicación controlada de cargas articulares y cambios volumétricos de flujo salival. Las mujeres tuvieron un FSE y FSNE menor al de los hombres. La aplicación de sobrecargas articulares por un breve período de tiempo provoca una caída del pH del FSE luego de 3 días de aplicación de cargas. Los resultados de esta investigación sugieren que el uso de aparatos intraorales por períodos cortos de tiempo no afecta el flujo salival. Los mecanismos neurofisiológicos involucrados en la mantención del equilibrio volumétrico de la saliva, pese a la estimulación de los mecanoreceptores, requiere mayor investigación.

\section{REFERENCIAS BIBLIOGRÁFICAS}

1. Yeh $K$, Hatchl $P$, Jonson A, Dodds J. Association of salivary flow rates with maximal bite force. J Dent Res, 2000; 79(8): 1560-1565.

2. Ikebe K, Matsuda K, Morii K, Tomohiro H. Relationship between bite force and salivary flow in older adults. Oral Surg Oral Med Oral Pathol Oral Radiol Endod, 2007; 104: 510-522.
3. Humphrey P, Russell T. A review of saliva: Normal composition, flow, and function. J Prosthet Dent, 2001; 85: 162-169.

4. Carpenter $\mathrm{H}$, Gordon $\mathrm{P}$. Regulation of salivary gland function by autonomic nerves. Autonomic Neuroscience: basic and clinical, 2007; 133: 3-18. 
5. Kogawa M, Calderón S, Lauris P, Araujo R, Conti R. Evaluation of maximal bite force in temporomandibular disorders patients. Journal of Oral Rehabilitation, 2006; 33: 559-565.

6. Poveda Roda R, Bagan JV, Díaz Fernández JM, Hernández Bazán S, Jiménez Soriano Y. Review of temporomandibular joint pathology. Part I: classification, epidemiology and risk factors. Med Oral Patol Oral Cir Bucal, 2007 Aug 1; 12(4): 292-298.

7. Fanghänel J, Gedrange T. On the development, morphology and function of the temporomandibular joint in the light of the orofacial system. Annals Anatomy, 2007; 189: 314-319.

8. Mese H, Matsuo R. Salivary secretion, taste and hyposalivation. Journal of Oral Rehabilitation, 2007; 34: 711-723.

9. De Almeida P del V, Grégio AM, Machado MA, de Lima AA, Azevedo LR. Saliva composition and functions: A comprehensive review. J Contemp Dent Pract, 2008 Mar 1; 9(3): 72-80.

10. Dworkin S, LeResche L. Research diagnostic criteria for temporo?mandibular disorders: Review, criteria, examinations and specifications, critique. J Craniomandib Disord, 1992; 6: 301-355.

11. Seedorf H, Scholz A, Kirsch I, Fenske C, Jüde D. Pivot appliances - is there a distractive effect on the temporomandibular joint? Journal of Oral Rehabilitation, 2007; 34: 34-40.

12. Poster W, Gebrian B, Ralph V, Spielman A. Effect of childhood malnutrition on salivary flow and pH. Archives of Oral Biology, 2008; 53: 231-237.
13. Bratthall D, Hänsel Petersson G, Stjernswärd JR. Cariogram Software, Internet Version 2.01. http://www.db.od.mah.se/car/cariogram/ cariograminfo.html. 2004.

14. Tschoppe P, Wolgin M, Pischon N, Kielbassa AM. Etiologic factors of hyposalivation and consequences for oral health. Quintessence Int, 2010 Apr; 41(4): 321-333.

15. Moen K, Bertelsen L, Hellem S, Jonsson R, Brun J. Salivary gland and temporomandibular joint involvement in rheumatoid arthritis: Relation to disease activity. Oral Diseases, 2005; 11: 27-34.

16. Scott J, Bajaj J, Linden R. The contribution of mechanoreceptive neurons in the gingival tissues to the masticatory - parotid salivary reflex in man. Journal of Rehabilitation, 1999; 26: 791-797.

17. Gaviäo M, Engelen L, van der Bilt A. Chewing behavior and salivary secretion. Eur J Oral Sci, 2004; 112: 19-24.

18. Anderson D, Hector $P$, Linden W. The possible relation between mastication and parotid secretion in the rabbit. J Physil, 1984; 364: 19-29.

19. Ikebe K, Hazeyama T, Iwase K, Sajima H, Gonda T, Maeda Y, Nokubi T. Association of symptomless TMJ sounds with occlusal force and masticatory performance in older adults. Journal of Oral Rehabilitation, 2008; 35: 317-323. 